\title{
Preoperative difficult airway prediction using suprahyoid and infrahyoid ultrasonography derived measurements in anesthesiology
}

\author{
Cristina Petrișor, Dan Dîrzu, Sebastian Trancă, Natalia Hagău, Constantin Bodolea
}

Anaesthesia and Intensive Care 2 ${ }^{\text {nd }}$ Department, "Iuliu Haţieganu” University of Medicine and Pharmacy Cluj-Napoca, Romania

\begin{abstract}
Airway management is one of the most important skills in everyday practice of anesthesia. Improper airway management might contribute to significant morbidity and mortality. In some patients, clinical parameters do not anticipate all difficulties related to airway management. Ultrasonography (US) might confer a potential screening tool for difficult airway. Suprahyoid and infrahyoid US measurements have been investigated for difficult airway prediction in anesthesiology. The most extensively investigated parameter was the anterior neck soft tissue thickness measured at different levels: anterior to the hyoid bone, epiglottis and vocal cords commissure. Hyomental distances measured with the head placed in neutral, sniffing or maximal hyperextended position and the derived hyomental distance ratios have also been evaluated for difficult airway prediction. For the evaluation of the tongue, measurements such as thickness, cross-sectional area, width, volume and tongue-to-oral cavity ratio can be used. Thus, anesthesiologists have many available potential US measurements, which could provide information regarding airway anatomy during the preoperative airway assessment and could serve as potential screening parameters for difficult airway. Still, we do not yet know which of these provides optimal predictive accuracy and larger sample size studies are required to validate their use in the preoperative evaluation of the airway.
\end{abstract}

Keywords: ultrasonography; airway; difficult intubation

\section{Introduction}

Predicting difficult intubations by evaluating the airways is vital in everyday anesthesia practice. Inaccurate assessment may place the patient at risk of hypoxic events and even death if complications occur and appropriate ventilation cannot be maintained. There are several clinical predictors for difficult airway laryngoscopy or intubation: Mallampati score, the upper-lip bite test, interincisor distance, neck hyperextension, thyromental distance, sternomental distance, short neck, abnormal teeth, history of obstructive sleep apnea and neck cir-

Received 17.10.2018 Accepted 06.12.2018

Med Ultrason

2019, Vol. 21, No 1, 83-88

Corresponding author: Cristina Petrișor

Department of Anaesthesia and Intensive Care Clinical Emergency County Hospital of Cluj 400006 Clinicilor 3-5, Cluj-Napoca, Romania Phone/Fax: +40 264599438

E-mail: petrisor.cristina@umfcluj.ro cumference. These have poor to modest sensitivity and specificity in difficult airway prediction $[1,2]$.

Ultrasound (US) is a powerful new point-of-care tool for airway management and is widely available in the operating rooms, emergency departments and critical care units [3]. The technique is non-irradiating, reproducible, repeatable, inexpensive, non-time consuming and easy to perform $[3,4]$. US of the upper airway provides detailed anatomic information and has numerous potential clinical applications $[5,6]$. It can be used to estimate tracheal tube size, confirm correct tracheal tube and laryngeal mask placement, diagnose upper airway pathology, guide percutaneous tracheostomy or cricothyroidotomy and predict post-extubation stridor [7]. The role of US in anesthesia-related airway assessment is encouraging although it is poorly defined. The main advantage of US in anesthesia seems to be related to its predictive power for difficult airway [8]. Several types of measurements have been investigated aiming to find a simple and reliable parameter which identifies patients with a difficult airway and thus helps the clinician in choosing the adequate in- 
tubation sequence, conferring safer airway management. Measurements such as anterior neck soft tissue thickness (ANS), tongue measurements, hyomental distances and composite indexes have been assessed.

In 2003 Ezri et al published a paper on difficult airway prediction based on the US measurement of the ANS [9]. By 2016 several studies have suggested that US, could offer several measurements for difficult airway prediction $[3,10]$. The advantages of US and the availability of ultrasound machines have boosted clinical interest in preoperative predictive US, especially in the operating room. However, in 2016 and 2017, concerns have been raised regarding the ability of US measurements to predict difficult airway as no single measurement or parameter could confer optimal sensitivity and specificity $[11,12]$. Recent results from a meta-analysis based on eight original studies concluded that the pooled characteristics of US-derived measurements predict difficult airway with $69 \%$ sensitivity and $84 \%$ specificity, comparable to those of computed tomography and X-ray [13]. Nonetheless, recent studies on the diagnostic accuracy of US-derived measurements for difficult airway have emerged, studies that continued to highlight the advantages of this technique (Table I). Airway US might constitute a useful tool provided simple scanning algorithms are established. In fact, US could become a first-line non-invasive airway assessment tool in the future [5]

The aim of this educational paper is to describe suprahyoid and infrahyoid US measurements investigated so far and summarize the current knowledge regarding their diagnostic performance for difficult airway detection.

Which ultrasound-derived parameters could serve as potential screening tools for difficult airway?

Several US measurements have been evaluated. The most studied was ANS at different levels. Other parameters were tongue thickness and tongue-to-oral cavity ratio, hyomental distances with derived ratios and composite scores. All studies excluded pregnant patients, patients with airway tumors, distorted airways, exter- nal laryngeal manipulation and laryngeal mask insertion. Optimal sniffing position, good muscle relaxation and an experienced laryngoscopist are required to allow comparisons between studies [26]. The reference standard is the Cormack-Lehane scale, with grades III and IV representing a difficult view of the larynx during direct laryngoscopy [27]. This is not synonymous with difficult intubation, but definitely confers homogeneity for studies on difficult airway prediction.

By means of US in the suprahyoid region it is possible to investigate the submandibular and sublingual spaces, the floor of the mouth and the root of the tongue $[28,29]$. Hyomental distances (HMD) can be measured with the head placed in neutral, sniffing or maximal hyperextended position and the derived hyomental distance ratios (HMDR) can then be calculated. For the evaluation of the tongue, measurements such as thickness, cross-sectional area, width, volume, tongue-to-oral cavity ratio can be measured. In the infrahyoid region, the visceral space includes the thyroid gland, the larynx and hypopharynx, as well as the cervical trachea [29-31]. The soft tissue thickness of the neck can be measured at different levels: anterior to the hyoid bone, epiglottis and vocal cords commissure.

\section{Anterior neck soft tissue thickness}

ANS has been measured in obese and non-obese patients. Measurements were taken at the level of the hyoid bone, through the thyrohyoid membrane, to the epiglottis and at the level of the vocal cords.

In order to evaluate ANS, the described scanning technique implies the measurement of the distances between the skin and three different anatomical structures. The patient is positioned with the head in neutral position and the linear transducer is placed perpendicular to the laryngeal axis. The anterior border of the hyoid bone is visualized and appears hyperechoic (fig 1a), while the epiglottis appears hypoechoic. Through the thyrohyoid membrane, the epiglottis appears curvilinear and its posterior border is demarcated by the air-mucosal interface

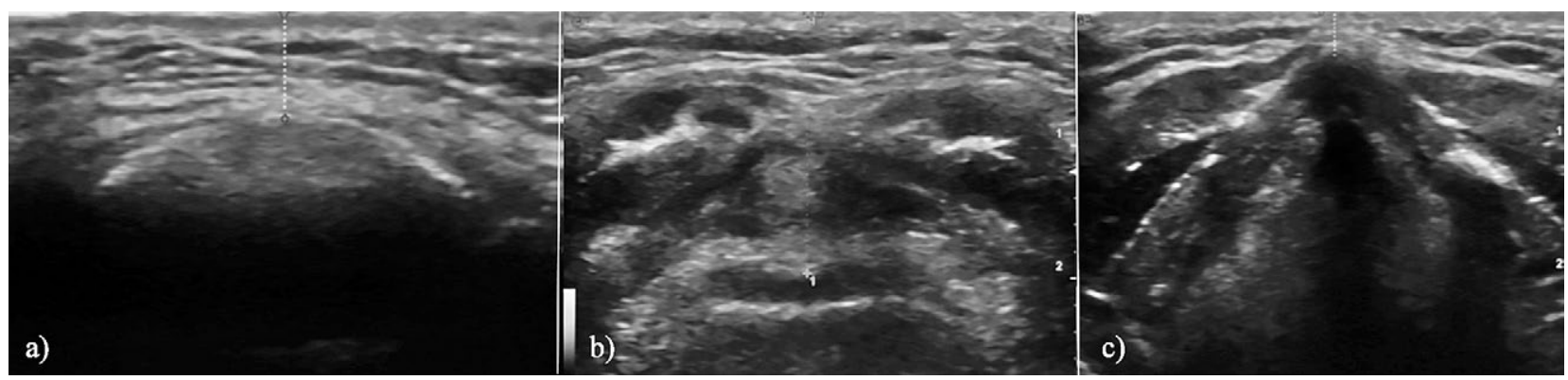

Fig 1. Anterior soft tissue thickness measured at the level of the hyoid (a), pre-epiglottic (b) and the anterior commissure of the vocal cords (c) 
Table I. Diagnostic accuracy of different investigated suprahyoid and infrahyoid ultrasound measurements for difficult airway prediction

\begin{tabular}{|c|c|c|c|c|c|}
\hline Reference & Measurement & $\mathbf{N}$ & $\mathrm{Se} \%$ & Sp\% & Cutoff \\
\hline [9] & ANS- vocal cords & 50 (obese) & \multicolumn{3}{|c|}{$\begin{array}{l}\text { Good discriminator between easy } \\
\text { and difficult laryngoscopy }\end{array}$} \\
\hline$[14]$ & ANS- vocal cords & 64 (obese) & \multicolumn{3}{|c|}{$\begin{array}{l}\text { Does not discriminate between } \\
\text { easy and difficult laryngoscopy }\end{array}$} \\
\hline [15] & $\begin{array}{l}\text { ANS- hyoid } \\
\text { ANS- pre-epiglottic }\end{array}$ & 51 & \multicolumn{3}{|c|}{ Not assessed } \\
\hline [16] & HMDR & 12 (obese) & \multicolumn{3}{|c|}{$\begin{array}{l}\text { Good discriminator between easy } \\
\text { and difficult laryngoscopy }\end{array}$} \\
\hline [17] & Sublingual ultrasound & 110 & \multicolumn{3}{|c|}{ Inability to see the hyoid bone } \\
\hline$[18]$ & $\begin{array}{l}\text { ANS- hyoid } \\
\text { ANS- pre-epiglottic } \\
\text { ANS- vocal cords }\end{array}$ & 203 & $\begin{array}{l}85.7 \\
100 \\
75\end{array}$ & $\begin{array}{l}85.1 \\
66.3 \\
80.6\end{array}$ & $\begin{array}{l}1.28 \mathrm{~cm} \\
1.78 \mathrm{~cm} \\
1.1 \mathrm{~cm}\end{array}$ \\
\hline [19] & ANS- pre-epiglottic & 74 & 64.7 & 77.1 & $2.75 \mathrm{~cm}$ \\
\hline$[11]$ & $\begin{array}{l}\text { HMDR max/neutral } \\
\text { Tongue thickness, volume, cross-sectional area, width, } \\
\text { tongue-to-oral cavity ratio, floor of the mouth muscle area } \\
\text { and volume }\end{array}$ & 199 & $\begin{array}{l}42.9 \\
9.1-31.7\end{array}$ & $68.4-97$ & \\
\hline$[20]$ & $\mathrm{PE} / \mathrm{P}-\mathrm{VC}$ & 53 & 87.5 & 30 & \\
\hline$[21]$ & $\begin{array}{l}\text { Tongue thickness } \\
\text { Tongue thickness to thyromental distance ratio }\end{array}$ & 2254 & $\begin{array}{l}75 \% \\
84 \%\end{array}$ & $\begin{array}{l}72 \\
79\end{array}$ & $\begin{array}{l}6.1 \mathrm{~cm} \\
>0.87\end{array}$ \\
\hline$[12]$ & $\begin{array}{l}\text { ANS-hyoid } \\
\text { ANS-preepiglottic } \\
\text { Width of the tongue, tongue volume, } \\
\text { floor of the mouth cross-sectional area }\end{array}$ & 130 & $\begin{array}{l}58.3 \\
75 \\
50-66.7\end{array}$ & $\begin{array}{l}56.8 \\
63.6 \\
55.9-62.7\end{array}$ & \\
\hline [22] & $\begin{array}{l}\text { ANS- pre-epiglottic } \\
\text { ANS- vocal cords }\end{array}$ & 301 & $\begin{array}{l}82 \\
\mathrm{NC}\end{array}$ & 91 & $0.254 \mathrm{~cm}$ \\
\hline$[7]$ & $\begin{array}{l}\text { ANS- hyoid } \\
\text { ANS- vocal cords } \\
\text { PE/E-VC }\end{array}$ & 100 & $\begin{array}{l}\text { NI85.7 } \\
\text { NI }\end{array}$ & 86.7 & $0.23 \mathrm{~cm}$ \\
\hline [23] & $\begin{array}{l}\text { HMDR max/neutral } \\
\text { HMDR sniffing/neutral } \\
\text { ANS- pre-epyglottic }\end{array}$ & 25 (obese) & $\begin{array}{l}100 \\
75 \\
75\end{array}$ & $\begin{array}{l}90.5 \\
76.2 \\
75\end{array}$ & $\begin{array}{l}1.23 \\
1.12 \\
1.38 \mathrm{~cm}\end{array}$ \\
\hline$[24]$ & $\begin{array}{l}\text { HMDR max/neutral } \\
\text { HMDR sniffing/neutral } \\
\text { ANS- pre-epiglottic }\end{array}$ & 120 & $\begin{array}{l}86 \\
57 \\
57.14\end{array}$ & $\begin{array}{l}72 \\
93 \\
91.82\end{array}$ & $\begin{array}{l}1.24 \\
1.06 \\
1.9 \mathrm{~cm}\end{array}$ \\
\hline$[25]$ & $\begin{array}{l}\text { HMDR max/neutral } \\
\text { PE/E-VC }\end{array}$ & 120 & $\begin{array}{l}75 \\
82\end{array}$ & $\begin{array}{l}85.3 \\
80\end{array}$ & $\begin{array}{l}1.085 \\
1.77\end{array}$ \\
\hline
\end{tabular}

$\mathrm{N}=$ number of patients; $\mathrm{Se} \%=$ sensitivity; $\mathrm{Sp} \%=$ specificity; $\mathrm{US}=$ ultrasound; ANS = anterior neck soft tissue thickness; HMDR = hyomental distance ratio; $\mathrm{PE} / \mathrm{E}-\mathrm{VC}=$ the ratio of the pre-epiglottic space to the distance from the epiglottis to the midpoint between the vocal cords. NC - No correlation; NI - Not indicative

(fig 1b). In this plane, the anterior commissure and the vocal cords are also visible and appear as mobile hyperechoic structures (fig 1c).

Ezri et al have noticed that in obese patients the ANS measured at the level of the vocal cords discriminated between patients with easy versus difficult intubation [9]. A similar study conducted on obese patients, but from a different geographical population, did not confirm this method and could not differentiate the patients with easy versus difficult intubation [14]. In a pilot study we conducted on 25 morbidly obese patients, the sensitivity and specificity of the method were $75 \%$ [23].

In the non-obese patients ANS measurements at the levels of the hyoid bone and epiglottis might discriminate between easy and difficult laryngoscopy [15]. Reported sensitivities and specificities for ANS measured at different levels vary considerably among studies $[7,12,18,19,22,24]$. These clinical studies are not fully 
comparable and each includes only modest numbers of patients. However, most of them suggest that the ANS distances are independent predictors of a difficult laryngoscopy [18]. ANS at different levels might be related to airway mobility during laryngoscopy. The hypothesis is that fat pads influence the view during direct laryngoscopy [9]. The abundance of pretracheal soft tissue might be a good predictor of difficult laryngoscopy as the mobility of the pharyngeal structures is impaired.

\section{Tongue measurements}

Tongue thickness and width, cross-sectional area and volume, as well as tongue thickness-to-oral cavity ratio are easily measured by placing a curvilinear ultrasound probe in the submental region in either mid-sagittal or transverse planes (fig 2).

In the studies of Andruszkiewicz et al [11] and Parameswari et al [12], tongue measurements such as thickness, volume and width, together with volume and tongue-to-oral cavity ratio have been shown to provide low to modest sensitivities. These led the authors to conclude that their use as screening parameters is inappropriate. Similarly, the US evaluation of the floor of the mouth muscles has low accuracy [11]. However, tongue thickness and tongue thickness-to-thyromental distance ratios provided higher sensitivities when further studied in a subsequent large-sample study published by Yao et al, concluding that these measurements have the optimal diagnostic accuracy [21].

Macroglosia is listed as a clinical predictor for difficult airway in all anesthesia textbooks as it does not allow good visualization of the larynx during direct laryngoscopy. The only three available clinical studies which investigated tongue thickness, provide contradictory results $[11,12,21]$. The study of Yao et al has the highest number of patients and concluded that these parameters have the potential to predict difficult tracheal intubation [21].

\section{Hyomental distances and derived ratios}

HMD and HMDR can be measured with the help of US by placing a curvilinear probe in the midsagittal position in the submental area. The investigator can easily identify the bright hyperechoic structures: the mandible and the hyoid bone. HMD is measured between the posterior aspect of the symphisis menti and the anterior border of the hyoid (fig 2).

HMD in maximal hyperextended position and HMDR maximal/neutral (the ratio obtained by dividing the HMD measured with the head fully extended to that in neutral position) have been identified as discriminative markers between obese patients with easy versus difficult laryngoscopy [16]. Andruszkiewicz et al have found a modest sensitivity of $42.9 \%$ for difficult airways [11]. Recently,

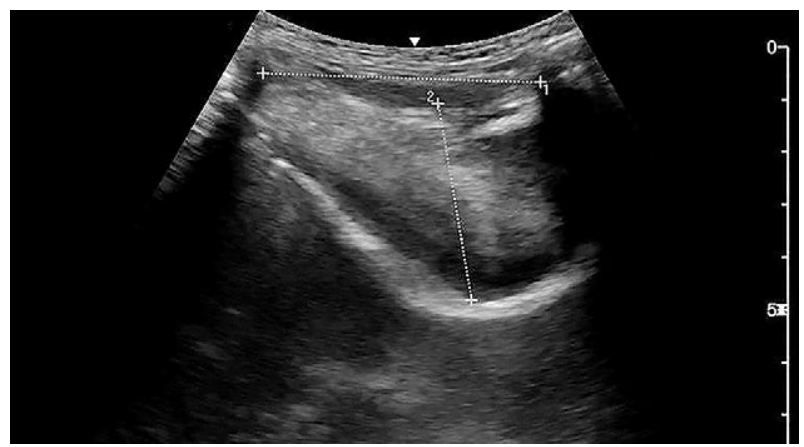

Fig 2. Hyomental distance and tongue thickness measured with the curvilinear transducer in sagital plane in the submandibular region

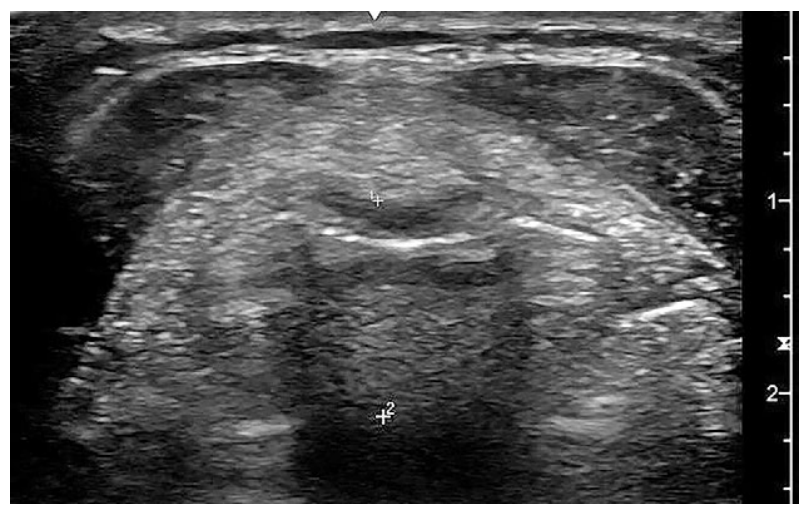

Fig 3. The pre-epiglottic distance and the distance between the epiglottis and the midpoint between the vocal cords, measured with a linear transducer that is rotated in a transverse plane from cephalad to caudal direction.

in three published studies, sensitivities have been appreciated to be higher $[23,24,25]$.

For HMDR sniffing/neutral (the HMDR obtained by dividing HMD in standard sniffing position for anesthesia induction to that in neutral position) the diagnostic accuracy compared to HMDR maximal/neutral was found to be lower [23,24]. Thus, HMDR maximal/neutral seems to be more accurate to predict a difficult airway.

HMDs and HMDR obtained by dividing the hyomental distance measured in maximal hyperextension to that in neutral position, measured clinically, have been shown to have predictive roles for a difficult airway [32]. HMDR is related to the ability of achieving good neck extension. The hyoid has a fixed position in relation to the base of the skull. The stylohyoid ligament stationary fixes the hyoid bone to the occiput [16]. With the head maximally extended, the mandible is moved away from the hyoid, which is relatively fixed in position. In patients with limited submandibular space compliance, visualization of the larynx can become difficult because the HMD does not expand sufficiently. Thus, HMD measurements 
reflect the elasticity in the sagittal plane. Wojtczak et al described a point-of-care simple scanning algorithm that could be easily used in anesthesia, emergency and intensive care practice [33]. Up to now, the studies investigating the performance of HMDR maximal/neutral for difficult airway prediction included only a modest number of patients. The reported sensitivities and specificities identified in most of them suggested that this easy-to-measure parameter might be useful for difficult airway prediction. However, larger sample studies are awaited.

\section{Pre-epiglottic distance divided by the distance between the epiglottis and the midpoint between the vocal cords $(P E / E-V C)$}

The ratio of the pre-epiglottic distance to the distance between the epiglottis and the midpoint of the vocal cords $(\mathrm{PE} / \mathrm{E}-\mathrm{VC})$ are calculated parameters. These are measured with a linear transducer that is placed in transverse plane and angled from cephalad to caudal until the epiglottis, the posterior parts of the vocal cords and the arytenoid cartilages are visible in a single oblique transverse plane $[7,34]$. The epiglottis is seen as a curvilinear hypoechoic structure and the vocal cords appear as hyperechoic lateral V-shaped structures (fig 3).

Reported sensitivity for PE/E-VC was $82-87.5 \%$, while specificity varied widely between 30 and $80 \%$ $[20,25]$. Reddy et al concluded that this parameter is not indicative for difficult intubation [7]. Gupta et al showed that PE/E-VC strongly correlates with the Cormack-Lehane grading. The grade can be predicted with a 67 to $68 \%$ sensitivity [34].

These studies investigating PE/E-VC have included modest numbers of patients and have contradictory results $[7,20,25]$. Further adequately powered studies are necessary to establish its use in anesthesiology practice.

\section{Sublingual ultrasound}

A unique US-based method to assess difficult airway is sublingual US. The inability to visualize the hyoid bone suggests a difficult airway [17].

\section{Conclusions}

Anesthesiologists have many available US-derived parameters, which could provide additional information regarding airway anatomy during the preoperative airway evaluation. These could serve as potential screening parameters for a difficult laryngoscopy/difficult airway. Still, we do not yet know which of these provides the optimal predictive accuracy.

In the future, larger sample size studies are required to compare the numerous ultrasound parameters' performances and to validate their use in the preoperative evaluation of the airway.

\section{References}

1. Shiga T, Wajima ZI, Inoue T, Sakamoto A. Predicting Difficult Intubation in Apparently Normal Patients. A Metaanalysis of Bedside Screening Test Performance. Anesthesiology 2005;103:429-437.

2. Roth D, Pace NL, Lee A, et al. Airway physical examination tests for detection of difficult airway management in apparently normal adult patients. Cochrane Database Syst Rev 2018;5:CD008874.

3. Teoh WH, Kristensen MS. Utility of ultrasound in airway management. Trends Anaesth Crit Care 2014;4:84-90.

4. Šustic A. Role of ultrasound in the airway management of critically ill patients. Crit Care Med 2007;35:S173-S177.

5. Osman A, Sum KM. Role of upper airway ultrasound in airway management. J Intensive Care 2016;4:52.

6. Singh M, Chin KJ, Chan VW, Wong DT, Prasad GA, Yu E. Use of sonography for airway assessment. J Ultrasound Med 2010;29:79-85.

7. Reddy PB, Punetha P, Chalam KS. Ultrasonographya viable tool for airway assessment. Indian $\mathrm{J}$ Anaesth 2016;60:807-813.

8. Kundra P, Mishra SK, Ramesh A. Ultrasound of the airway. Indian J Anaesth 2011;55:456-462.

9. Ezri T, Gewürtz G, Sessler DI, et al. Prediction of difficult laryngoscopy in obese patients by ultrasound quantification of anterior neck soft tissue. Anaesthesia 2003;58:11111114.

10. Fulkerson JS, Moore HM, Anderson TS, Lowe RF Jr. Ultrasonography in the preoperative difficult airway assessment. J Clin Monit Comput 2017;31:513-530.

11. Andruszkiewicz P, Wojtczak J, Sobczyk D, Stach O, Kowalik I. Effectiveness and validity of sonographic upper airway evaluation to predict difficult laryngoscopy. J Ultrasound Med 2016;35:2243-2252.

12. Parameswari A, Govind M, Vakamudi M. Correlation between preoperative ultrasonographic airway assessment and laryngoscopic view in adult patients: a prospective study. J Anaesth Clin Pharmacol 2017;33:353-358.

13. Ji C, Ni Q, Chen W. Diagnostic accuracy of radiology (CT, $\mathrm{X}$-ray, US) for predicting difficult intubation in adults: A meta-analysis. J Clin Anesth 2018;45:79-87.

14. Komatsu R, Sengupta P, Wadhwa A, et al. Ultrasound quantification of anterior soft tissue thickness fails to predict difficult laryngoscopy in obese patients. Anaesth Intensive Care 2007;35:32-37.

15. Adhikari S, Zeger W, Schmier C, et al. Pilot Study to Determine the Utility of Point-of-care Ultrasound in the Assessment of Difficult Laryngoscopy. Acad Emerg Med 2011;18:754-758.

16. Wojtczak JA. Submandibular sonography: assessment of hyomental distances and ratio, tongue size, and floor of the mouth musculature using portable sonography. J Ultrasound Med 2012;31:523-528.

17. Hui CM, Tsui BC. Sublingual ultrasound as an assessment method for predicting difficult intubation: a pilot study. Anaesthesia 2014;69:314-319. 
18. Wu J, Dong J, Ding Y, Zheng J. Role of anterior neck soft tissue quantifications by ultrasound in predicting difficult laryngoscopy. Med Sci Monit 2014;20:2343-2350.

19. Pinto J, Cordeiro L, Pereira C, Gama R, Fernandes HL, Assunção J. Predicting difficult laryngoscopy using ultrasound measurement of distance from skin to epiglottis. JCritical Care 2016;33:26-31.

20. Soltani Mohammadi S, Saliminia A, Nejatifard N, Azma R. Usefulness of Ultrasound View of Larynx in Pre-Anesthetic Airway Assessment: A Comparison With Cormack-Lehane Classification During Direct Laryngoscopy. Anesth Pain Med 2016;6:e39566.

21. Yao W, Wang B. Can tongue thickness measured by ultrasonography predict difficult tracheal intubation? $\mathrm{Br} \mathrm{J}$ Anaesth 2017;118:601-609.

22. Falcetta S, Cavallo S, Gabbanelli V, et al. Evaluation of two neck ultrasound measurements as predictors of difficult direct laryngoscopy: A prospective observational study. Eur J Anaesthesiol 2018;35:605-612.

23. Petrisor C, Szabo R, Constantinescu C, Prie A, Hagau N. Ultrasound-based assessment of hyomental distances in neutral, ramped, and maximum hyperextended positions, and derived ratios, for the prediction of difficult airway in the obese population: a pilot diagnostic accuracy study. Anaesthesiol Intensive Ther 2018;50:110-116.

24. Petrisor C, Szabo R, Constantinescu C, Prie A, Tranca S, Hagau N. The performance of ultrasound-based quantification of the hyomental distance ratio in predicting difficult airway in anaesthesia: A STARD-compliant prospective diagnostic study. Eur J Anaesthesiol 2018;35:627-628.

25. Rana S, Verma V, Bhandari S, Sharma S, Koundal V, Chaudhary SK. Point-of-care ultrasound in the airway as- sessment: A correlation of ultrasonography-guided parameters to the Cormack-Lehane Classification. Saudi J Anaesth 2018;12:292-296.

26. Xue FS, Sun C, Liu GP, Yang GZ. Predicting difficult laryngoscopy using ultrasound technique. J Critical Care 2016;34:131-132.

27. Cormack RS, Lehane J. Difficult tracheal intubation in obstetrics. Anaesthesia 1984;39:1105-1111.

28. Gervasio A, d'Orta G, Mujahed I, Biasio A. Sonographic anatomy of the neck: the suprahyoid region. J Ultrasound 2011;14:130-135.

29. Choudhary G, Boparai A, Singh G, Singh R. Value of ultrasound in understanding laryngeal anatomy: pictorial review. Pakistan J Radiology 2012;22:4-7.

30. Gervasio A, Mujahed I, Biasio A, Alessi S. Ultrasound anatomy of the neck: the infrahyoid region. J Ultrasound 2010;13:85-89.

31. Costache A, Dumitru M, Anghel I, Cergan R, Anghel AG, Sarafoleanu C. Ultrasonographic anatomy of head and neck-a pictorial for the ENT specialist. Med Ultrason 2015;17:104-108.

32. Huh J, Shin HY, Kim SH, Yoon TK, Kim DK. Diagnostic predictor of difficult laryngoscopy: the hyomental distance ratio. Anesth Analg 2009;108:544-548.

33. Wojtczak J, Bonadonna P. Pocket mobile smartphone system for the point-of-care submandibular ultrasonography. Am J Emerg Med 2013;31:573-577.

34. Gupta DE, Srirajakalidindi AR, Ittiara BR, Apple LE, Toshniwal GO, Haber HA. Ultrasonographic modification of Cormack Lehane classification for pre-anesthetic airway assessment. Middle East J Anaesthesiol 2012;21:835842 . 